\title{
Temporal ChIP-on-chip reveals Biniou as a universal regulator of the visceral muscle transcriptional network
}

\author{
Janus S. Jakobsen, ${ }^{1}$ Martina Braun, ${ }^{1}$ Jeanette Astorga, ${ }^{2}$ E. Hilary Gustafson, ${ }^{1}$ Thomas Sandmann, ${ }^{1}$ \\ Michal Karzynski, ${ }^{1}$ Peter Carlsson, ${ }^{2}$ and Eileen E.M. Furlong ${ }^{1,3}$ \\ ${ }^{1}$ European Molecular Biology Laboratory, D-69117 Heidelberg, Germany; ${ }^{2}$ Department of Cell and Molecular Biology, \\ Goteborg University, SE-405 30 Goteborg, Sweden
}

Smooth muscle plays a prominent role in many fundamental processes and diseases, yet our understanding of the transcriptional network regulating its development is very limited. The FoxF transcription factors are essential for visceral smooth muscle development in diverse species, although their direct regulatory role remains elusive. We present a transcriptional map of Biniou (a FoxF transcription factor) and Bagpipe (an Nkx factor) activity, as a first step to deciphering the developmental program regulating Drosophila visceral muscle development. A time course of chromatin immunoprecipitatation followed by microarray analysis (ChIP-on-chip) experiments and expression profiling of mutant embryos reveal a dynamic map of in vivo bound enhancers and direct target genes. While Biniou is broadly expressed, it regulates enhancers driving temporally and spatially restricted expression. In vivo reporter assays indicate that the timing of Biniou binding is a key trigger for the time span of enhancer activity. Although bagpipe and biniou mutants phenocopy each other, their regulatory potential is quite different. This network architecture was not apparent from genetic studies, and highlights Biniou as a universal regulator in all visceral muscle, regardless of its developmental origin or subsequent function. The regulatory connection of a number of Biniou target genes is conserved in mice, suggesting an ancient wiring of this developmental program.

[Keywords: Biniou; Bagpipe; visceral muscle; ChIP-on-chip; transcriptional network; temporal regulation]

Supplemental material is available at http://www.genesdev.org.

Received April 19, 2007; revised version accepted August 7, 2007.

Genetic and molecular studies have made substantial progress in identifying key regulators essential for the specification of tissue primordia. Yet relatively little is known about the regulatory networks governing their subsequent differentiation into functional tissues. This is particularly true for the smooth muscle. In contrast to cardiac and skeletal muscle development, where the regulatory connectivity of multiple transcription factor (TF) families have been intensively studied (Pax3, MyoD, Mef2, BMP, Nkx, GATA, to name a few) (Doevendans and van Bilsen 1996; Black and Olson 1998; Baylies and Michelson 2001; Lamey et al. 2004; Berkes and Tapscott 2005), the transcriptional network governing visceral smooth muscle development remains largely uncharacterized.

Vertebrate visceral muscle (VM) consists of diverse muscle types that develop from different embryonic origins, and perform various functions ranging from blood

${ }^{3}$ Corresponding author.

E-MAIL furlong@embl.de; FAX 49-6221-387166.

Article is online at http://www.genesdev.org/cgi/doi/10.1101/gad.437607. vessel constriction and urogenital tract contraction, to the peristaltic movement of the gut. As a consequence, smooth muscle defects contribute to a host of human diseases including hypertension, asthma, and diseases affecting the gut connective tissue. In invertebrates, the situation is somewhat simpler. Drosophila embryos have four types of VM that all contribute to the gut musculature (for review, see Lee et al. 2005). Despite their different embryological origins, all VM in flies and humans display slow supercontractions, distinguishing them from skeletal (somatic) and cardiac muscle (Goldstein and Burdette 1971; Bitar 2003).

It is perhaps not surprising that a tissue with such fundamental function as moving food through the gut has many developmental and structural homologies across metazoans. What is more intriguing is the essential role of key regulators of VM development from flies to vertebrates. Mutations in FoxF TFs cause defects in gut muscle differentiation in both mice (Mahlapuu et al. 2001a; Wang et al. 2003; Ormestad et al. 2004, 2006) and Xenopus (Tseng et al. 2004), similar to those observed in flies (Zaffran et al. 2001). Likewise, BapX is required for 
the specification of subtypes of VM in mice (Lettice et al. 1999) and flies (Azpiazu and Frasch 1993). This high level of functional conservation suggests that the underlying transcriptional circuitry governing VM development may also be conserved.

To date, most progress has been made in elucidating the genetic program required for VM development in Drosophila (Kusch and Reuter 1999; San Martin and Bate 2001; Lee et al. 2005). The trunk VM is specified through the combined action of inductive signals from the overlying ectoderm ( $d p p$, a BMP family member) and intrinsic factors in the underlying splanchnic mesoderm (the TFs Tinman and sloppy-paired) (Frasch 1995; Riechmann et al. 1997; Lee and Frasch 2000). At the molecular level, the direct inputs from Dpp, Tinman and Sloppy-paired are integrated in a cis-regulatory module (CRM) that controls the expression of bagpipe, a BapX ortholog (Lee and Frasch 2005). Bagpipe, an Nkx TF, together with Dpp, regulates the expression of the FoxF TF, biniou (Zaffran et al. 2001).

Both Bagpipe and Biniou are essential for the specification of trunk VM. Embryos lacking either TF have a complete absence of circular midgut muscle (Azpiazu and Frasch 1993; Zaffran et al. 2001). Although the upstream regulators of both factors are well characterized, their downstream effectors are largely unknown. How do Bagpipe and Biniou regulate VM specification? Are these TFs only required for this process? The broad temporal and spatial expression of biniou suggests that it may be required to regulate other aspects, potentially all aspects, of the VM developmental program. However, there are currently too few characterized target genes and CRMs known to make accurate models about Biniou's direct regulatory role; only three direct target genes have been identified to date. Biniou provides positive feedback regulation to bagpipe during trunk VM specification (Zaffran et al. 2001). The two other target genes, $d p p$ (Zaffran et al. 2001) and $\beta 3$-tubulin (Zaffran and Frasch 2002), are required for midgut morphogenesis (Mathies et al. 1994; Dettman et al. 1996). The lack of trunk VM in biniou mutant embryos has precluded a detailed understanding of its function during later stages of VM development.

To decipher the transcriptional program regulating VM development within its in vivo context, we have used two complementary global approaches to systematically identify VM CRMs and their associated target genes during a developmental time course. The integration of expression profiling of biniou mutant embryos with chromatin immunoprecipitation followed by microarray analysis (ChIP-on-chip) experiments produced a temporal map of Biniou activity, revealing dynamic enhancer occupancy in vivo. Transgenic reporter assays indicate that the timing of Biniou enhancer binding precisely matches the timing of enhancer activity. Integrating these data with in vivo binding of Bagpipe showed that Bagpipe targets a restricted subset of CRMs during the early stages of VM specification. These data provide an initial global map of the regulatory circuitry driving the progression of VM development in flies, and high- lights that at least some of the transcriptional wiring is conserved in mice.

\section{Results} A developmental time course of gene expression
during VM development

To identify genes genetically downstream from biniou, we compared the expression profiles of wild-type embryos to tightly stage matched biniou mutant embryos during a developmental time series. Biniou mutants lack circular VM, which normally covers the midgut, and have secondary defects in longitudinal VM (Zaffran et al. 2001). Pure populations of biniou homozygous mutant embryos were isolated from their balancer siblings using an automated embryo sorter and manually censored, as described previously (Furlong et al. 2001a,b). Five consecutive 1-h time periods were examined, covering the stages of VM specification, migration, and the initiation of terminal differentiation (Fig. 1A). An additional time period was analyzed at the end of embryogenesis (stage 16) to identify genes potentially involved in VM terminal differentiation and function. A complementary genetic background was also examined: embryos with ectopic biniou expression throughout the mesoderm (Fig. 1A).

These experiments identified 1164 genes with significant changes in gene expression at one or more developmental time points or conditions, with $\sim 11$ predicted false positives (Supplementary Fig. 1; Supplementary Table 1). Of these, 639 genes had reduced expression in biniou loss-of-function mutants (Supplementary Fig. 1, clusters 1 and 2). These include many genes specifically associated with VM development (e.g., bap, Fas3, Con, pnt, HLH54F, and hand), more generally expressed muscle genes (sns, Imd, $\beta 3$ tub60D), as well as 415 genes of unknown function (predicted CGs). In contrast, 525 genes had increased expression in biniou mutants, which may represent a secondary systemic response of the embryo to a block in VM development (Supplementary Fig. 1, clusters 3 and 4).

\section{Systematic mapping of Biniou-bound enhancers and direct target genes during embryogenesis}

The expression profiling identified a large number of previously uncharacterized genes as genetically downstream from biniou. We next set out to determine which genes are directly regulated by this TF and to identify the associated enhancer regions. ChIP-on-chip was performed against the endogenous Biniou protein during a developmental time course. Four consecutive 2-h time points were examined, covering the stages of visceral mesoderm induction to the initiation of terminal muscle differentiation (Fig. 1A, stages 10-15). To reduce the number of false positives due to possible nonspecific binding of the antiserum, two independent anti-Biniou antisera were used for the immunoprecipitations. These samples were hybridized to a tiling array consisting of overlapping 3 -kb fragments covering $~ 50 \%$ of the Dro- 

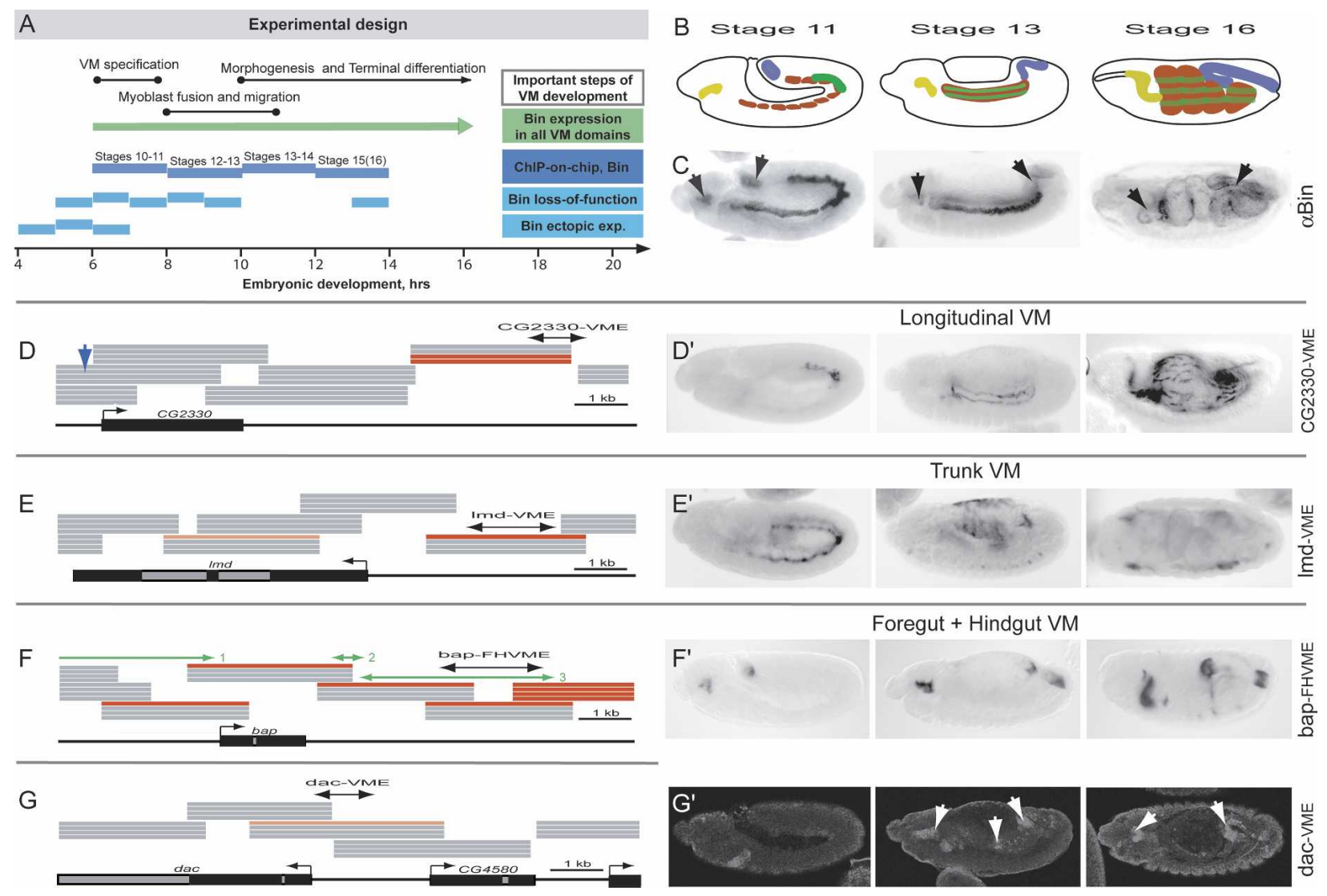

Figure 1. Biniou-bound enhancers drive expression in diverse spatial domains. $(A)$ Schematic diagram showing the experimental design. The green arrow indicates Biniou's continuous expression from stage $10(\sim 6 \mathrm{~h}$ after egg laying) to the end of embryogenesis. ChIP-on-chip experiments were conducted at four consecutive 2-h time points (dark-blue bars). This was complemented by expression profiling of biniou loss-of-function and gain-of-function mutant embryos at 1-h time points, indicated by the light-blue bars. $(B)$ A schematic diagram of VM development showing the four different primordia that give rise to foregut (yellow), hindgut (purple), trunk (red), and longitudinal (green) VM during stages 11, 13, and 16 of embryogenesis (modified from Lee et al. 2006). (C) Immunostaining of Biniou in wild-type embryos at developmental stages 11,13, and 16. The foregut and hindgut VM are indicated by black arrows. $(D-G)$ Schematic overviews of four Biniou-bound regions used in transgenic reporter strains: The gene is depicted at the bottom of the panel, with exons in black and introns in gray. Genomic fragments on the tiling arrays are indicated as stacks of four horizontal gray bars in their corresponding genomic position. Each single bar represents the results from one ChIP-on-chip time period, with the earliest $(6-8 \mathrm{~h})$ positioned at the top of the stack and the latest $(12-14 \mathrm{~h})$ at the bottom of the stack (blue arrow in $D$ represents the direction of time). The red bars indicate Biniou binding, while light-red bars represent binding just below our significance cutoff. Black double-headed arrows mark the boundaries of the cloned regions. All enhancers are labeled by the target gene name, followed by VME (VM enhancer). The green double-headed arrows indicate the known enhancers of bagpipe. ( $\left.D^{\prime}-G^{\prime}\right)$ In situ hybridization of GFP transcripts in transgenic enhancer-GFP embryos for the regions indicated in $D-G$. The four enhancer lines drive specific expression in different subtypes of VM: longitudinal muscle (CG2330-VME) $\left(D^{\prime}\right)$, trunk VM (Imd-VME) (E'), and foregut and hindgut VM (bap-FH$\mathrm{VME})\left(F^{\prime}\right) .\left(G^{\prime}\right)$ Fluorescent in situ hybridization showing dac-VME expression in the trunk, foregut, and hindgut VM (white arrows). In $B-D$, embryos are oriented with the anterior to the left and the dorsal at the top. All views are lateral, except $E^{\prime}$ and $G^{\prime}$ stage 16 , which are dorsal.

sophila genome (Sandmann et al. 2006). Only regions that were significantly enriched using both antibodies were considered to be Biniou-bound enhancer regions.

Using these stringent criteria we identified 292 Biniou-bound regions at one or more developmental time points with a false discovery rate of $<1 \%$ (Supplementary Table 2). Due to the overlapping nature of the microarray, this represents 218 unique genomic regions. This set includes all known Biniou-bound enhancers, namely bagpipe (Lee and Frasch 2005), $\beta$-Tubulin at 60D (Zaffran and Frasch 2002), and dpp (Zaffran et al. 2001) enhancer regions (Supplementary Fig. 2).
As metazoan enhancers can work over long distances, and do not necessarily regulate the closest gene, connecting enhancer data to the correct target gene is very challenging. To link Biniou-bound regions to their putative target genes we used a systematic approach (Supplemental Material). In brief, a cumulative score was given to all genes in the vicinity of a Biniou-bound region based on (1) proximity; (2) requirement of biniou function for normal expression, determined by expression profiling of biniou loss-of-function mutant embryos described above; (3) the ability of ectopic Biniou to increase the gene's expression; and (4) spatial expression overlap- 
ping with Biniou (from BDGP in situ data and the literature). This method proved very effective in linking Mef2bound regions to their target genes (Sandmann et al. 2006). Using these criteria, 224 of the 292 bound regions were assigned to 146 direct target genes (Supplementary Table 3). This systematic approach correctly assigned the three known Biniou enhancers to their appropriate target gene, and also assigned a number of genes known to be genetically downstream from biniou (e.g., vimar, FasIII, bnl). All Biniou-bound regions and surrounding genes can be visualized at http://furlonglab.embl.de/data/ browse_chip_mod.

Biniou-bound regions drive expression in spatially restricted subdomains of the VM

The regulatory potential of four Biniou-bound regions was tested in vivo by examining their ability to drive GFP expression in transgenic animals. Conservation in other Drosophila species and the location of predicted Biniou motifs (Supplemental Material) were used as guides to select smaller regions within the Biniou-bound genomic fragments. All four regions were sufficient to function as enhancers in vivo, regulating expression in domains overlapping that of Biniou.

Biniou is expressed in all four subtypes of VM: the trunk VM and longitudinal VM surrounding the midgut, the foregut VM, and the hindgut VM (Fig. 1B). Although Biniou has a broad spatial expression (Fig. 1C), it regulates enhancers that drive expression in spatially restricted subtypes and subdomains of the VM. The 930base-pair (bp) enhancer region for CG2330 drives expression in the longitudinal VM, where it initiates expression soon after the onset of Biniou expression and continues to be expressed until the end of embryogenesis (Fig. $1 \mathrm{D}^{2} \mathrm{D}^{\prime}$ ). In contrast, the enhancer region for lmd drives expression in the trunk VM during early stages of development and is not expressed in VM later in development (Fig. 1E, $\mathrm{E}^{\prime}$ ). In the bagpipe locus, the previously identified bap3.5 enhancer (Lee and Frasch 2005) drives expression in both the foregut and hindgut visceral mesoderm (Fig. 1F, double-headed green arrow 3). Here, we show that this enhancer is bound by Biniou in vivo and report the boundaries of a smaller region (reduced from the previously reported $3.5 \mathrm{~kb}$ to $2.1 \mathrm{~kb}$ ) that is sufficient to drive expression in these two VM populations from stage 10 until the end of embryogenesis (Fig. $1 \mathrm{~F}^{\prime}$ ).

Biniou binding within the dachshund gene locus (Fig. 1G) was detected just below our stringent cutoff for enrichment ( $q$-value of 0.046 instead of $<0.01)$. Members of the dachs, eyes absent, and six family of transcriptional regulators are essential for skeletal muscle development in vertebrates. As dacs has no reported role in Drosophila muscle development it was of interest to examine the expression of this Biniou-bound region. This enhancer is sufficient to drive expression in the foregut and hindgut VM, in a group of cells in the central part of the trunk VM, as well as in the salivary glands (Fig. 1G', arrows). The observed expression is broader than that of the endogenous dac gene, which is limited to the hind- gut domain (Kumar and Moses 2001). This may reflect the absence of some negative regulatory inputs from the cloned enhancer region.

In summary, these data and those presented below demonstrate that our ChIP-on-chip results provide an accurate and sensitive global map of Biniou-bound enhancers, and that these regions drive expression in all four subtypes of VM.

\section{Biniou binds to three temporal groups of enhancers}

The broad temporal expression of Biniou (stages 10-17) suggests a regulatory role during all stages of VM development. The direct regulation of $\beta 3$-tub60D (Zaffran and Frasch 2002), a gene expressed continuously in the developing gut muscle, supports this model. However, Biniou also directly regulates bagpipe expression via the characterized bap3 enhancer (Lee and Frasch 2005). In contrast to $\beta 3$-tub60D, bagpipe is expressed in a narrow developmental window, indicating that Biniou can also regulate more transient gene expression. We used our ChIP-on-chip time course to obtain a global view of the temporal enhancer occupancy of Biniou during developmental progression.

K-medians clustering was used to group the 292 bound regions based on their levels of enrichment through developmental time. This analysis revealed three robust groups of temporally occupied enhancers (Fig. 2A; Supplementary Table 2). Almost half of the CRMs (47\%) are bound by Biniou at all four time points assayed, spanning stages 10-15 (Fig. 2A, continuously bound). This continuous enhancer binding mirrors the TF's continuous expression and suggests a requirement for Biniou to regulate the associated target genes during all stages of VM development.

Surprisingly, Biniou binds to two additional groups of enhancers in temporally distinct patterns. Twenty-two percent of enhancer regions are only bound by Biniou early in development (Fig. 2A, early-bound, stages 1011 ), and are not bound at later developmental stages (1415). In contrast, $31 \%$ of Biniou-bound enhancers are specifically bound at late stages in development, but not during the early stages of VM specification (Fig. 2A, latebound). As Biniou protein is expressed continuously in these cells, the early-bound and late-bound enhancers were unexpected and demonstrate that Biniou activity is regulated at the level of its enhancer binding.

\section{Biniou enhancer binding determines the timing of enhancer activity}

The dynamic enhancer binding suggests that the timing of Biniou occupancy is important for the timing of enhancer activity. To assess this in vivo, a number of regions from each temporal cluster (Fig. 2A) were linked to a GFP reporter. The timing of enhancer activity was assayed in vivo by in situ hybridization in transgenic embryos, to avoid time delays due to GFP protein folding and protein perdurance. All regions examined drive expression in a subset of Biniou-expressing cells and re- 
Jakobsen et al.
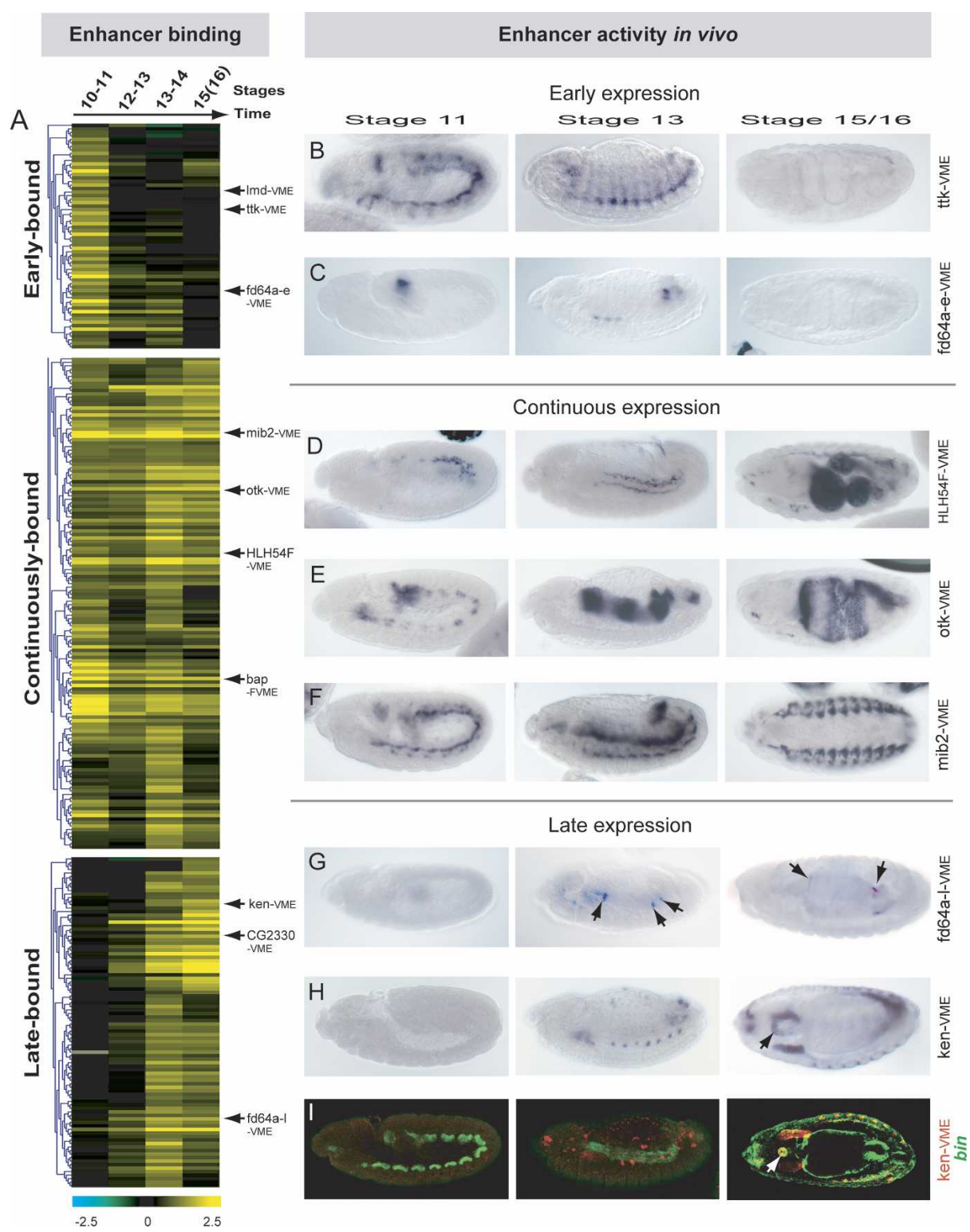

Figure 2. Dynamic enhancer binding. Biniou acts as a trigger for CRM activity. $(A)$ Biniou binds to CRMs in three temporal groups: $\mathrm{K}$-median clustering of the enrichment levels of Biniou binding in each consecutive time period uncovered three groups of enhancers bound either early, continuously, or late in development. Each row represents a Biniou-bound enhancer region, while each column represents one developmental time period, with stages 10-11 (6-8 h) to the left and stage 15 (12-14 h) to the right. The level of Biniou enrichment is displayed as a heat map, in which black reflects no enrichment and dark yellow represents high enrichment, quantified in the scale below the clustergram $\left(\log _{2}\right) .(B-I)$ The time span of Biniou binding correlates with enhancer activity: in situ hybridization of GFP transcripts in transgenic enhancer lines using enhancer regions from each temporal class in $A$. $(B)$ The enhancer in the $t t k$ locus is transiently expressed in the VM (stages 10-11) and somatic muscle (stages 11-13). (C) fd64a early enhancer drives expression in the hindgut VM from stages 11-13. $(D-F)$ Three continuously bound enhancers drive expression from stages 11-16 in the longitudinal VM (HLH54F-VME; D) or trunk VM (otk-VME; E). (F) mib-2 enhancer drives expression in the VM and somatic muscle from stages 11-13. Only somatic muscle expression is maintained at stage $16 .(G)$ The $f d 64 a$ late enhancer drives expression in a subset of the anterior and posterior region of the trunk VM from stages 13-15 (arrow). There is no early expression prior to the time point of Biniou binding. $(H)$ The ken enhancer is expressed in the salivary gland and neuronal tissue at stage 13 , and initiates expression in the foregut VM at stage 15 (arrow), matching the timing of Biniou binding. (I) Fluorescent in situ hybridization of biniou (green) and GFP (red) of the ken-VME showing coexpression in the foregut VM at stage 15 (white arrow). All embryos at stages 11 and 13 are lateral views. Stage 16 embryos in $B, C, E$, and $G$ are lateral views; $F$ is ventral; and $D, H$, and $I$ are dorsal views.

capitulate all or part of the target genes' expression (see Supplementary Fig. 3 for a detailed description). Here we focus on their temporal activity.
The initiation of enhancer activity closely matches the first time point of Biniou binding for $>90 \%$ of enhancers examined (10 of 11 CRMs) (Figs. 1, 2). The early- 
bound enhancers drive expression at stages 10-11, reflecting the binding of Biniou at these stages of development: $t$ tk enhancer (Fig. 2B), fd64a-e enhancer (Fig. 2C), Imd enhancer (Fig. 1E'), bap3 enhancer (Supplementary Table 2; Lee and Frasch 2005). Similarly, all four continuous-bound enhancers initiate expression at the first time period when Biniou binds (stages 10-11): HLH54F enhancer (Fig. 2D), otk enhancer (Fig. 2E), mib2 enhancer (Fig. 2F), bap-FH enhancer (Fig. 1F'). The two late-bound enhancers, on the other hand, do not initiate expression at stages 10 or 11 of development, matching the lack of Biniou binding during these stages (Fig. 2G,H, cf. A). Instead, the expression of the $f d 64 a$ late enhancer initiates at stage 13 (Fig. 2G, arrow), while the ken enhancer initiates VM expression at stage 14 (Fig. 2H,I, arrow). This shift in the initiation of activity mirrors Biniou binding to these enhancers at stages $12-13$ and 13-14, respectively (Fig. 2A). The only exception is the CG2330 enhancer, which initiates expression at stage 11, while Biniou enhancer binding was first detected at stage 1314 (Fig. 1D,D'). As the expression of endogenous CG2330 does not initiate until stage 13 (Supplementary Fig. 4), the apparent discrepancy in enhancer activity may simply reflect the exclusion of some regulatory motifs within the limits of the cloned region.

Remarkably, the duration of enhancer activity is also tightly correlated with the time span of Biniou binding in 10 out of 11 CRMs examined ( $90 \%)$. This is particularly striking in the early-bound enhancers: When Biniou ceases to bind to these CRMs, their ability to regulate expression is lost: Imd (Fig. 1E'), ttk (Fig. 2B), fd64a early (Fig. 2C), and bap3 (Supplementary Table 2). The converse is also true. Continuous Biniou binding correlates with continuous enhancer activity: bap-FH enhancer (Fig. $1 \mathrm{~F}^{\prime}$ ), HLH54F enhancer (Fig. 2D), and otk enhancer (Fig. 2E). The exception is the mib2 enhancer (Fig. 2F). In the context of this module Biniou binding it is not sufficient to maintain enhancer activity in the VM at late developmental time points.

Taken together, these data indicate that the timing of Biniou enhancer binding is predictive for temporal enhancer activity in the large majority of cases.

\section{Differential enrichment of TF motifs distinguish the three classes of Biniou-CRMs}

All 11 Biniou enhancers examined in vivo regulate expression in more restricted patterns than Biniou itself. As Biniou has broad temporal and spatial expression, additional regulatory inputs must refine Biniou's activity in a combinatorial manner. To identify other factors that may impinge on these enhancers we searched for overrepresented motifs within the Biniou-bound CRMs. This analysis identified significant enrichment of a number of TF motifs (Supplementary Fig. 5). Of particular interest is the differential enrichment of motifs for Biniou, Mef2, and Nkx family proteins, Bap and Tin, between the three temporal classes of enhancers (Fig. 3).

Interestingly, the Bap motif is specifically enriched in the early-bound enhancers, and not in the continuous- or

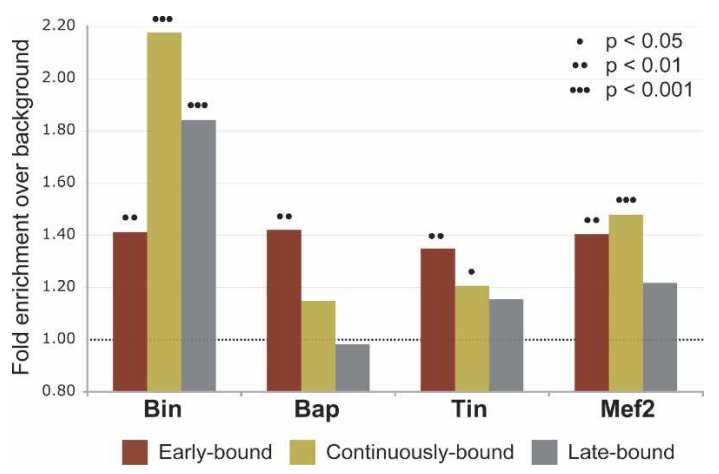

Figure 3. Differential enrichment of transcription motifs between the early-bound, continuously bound, and late-bound enhancers. The three temporal groups of enhancers (early-bound, continuously bound, and late-bound) are enriched in different TF motifs. The histogram shows the level of motif enrichment in the ChIP-bound enhancers compared with background ( $Y$-axis) for Bin, Bap, Tin, and Mef2 motifs. Motif enrichment is defined as the number of motifs per kilobase within the enhancers divided by the number of motifs per kilobase in random "background" DNA. Bap motifs are significantly overrepresented in the Biniou early-bound enhancers but not in the continuously or late-bound regions. Mef2 motifs are highly enriched in both the early-bound and continuously bound enhancers. While Tin motifs are strongly enriched in the early group, the continuous group has only a slight overrepresentation of this motif. The Biniou motif is enriched in all three groups, with particularly high enrichment in the continuously bound and late-bound enhancers. $(\bullet) P<0.05$; $(\bullet \bullet) P<0.01$; $(\bullet \bullet \bullet) P<0.001$.

late-bound group (Fig. 3). Tin motifs are also enriched in the early-bound group (Fig. 3). This is in agreement with the transient expression of both TFs in the trunk VM during early stages development (stages 10-11) (Azpiazu and Frasch 1993; Bodmer 1993), and suggests that one or both of these TFs could impart some of the specificity for Biniou transient binding to these enhancers.

The Mef2 motif is highly enriched in both early- and continuous-bound enhancers, but not in late VM enhancers (Fig. 3). This was surprising as Mef2 regulates muscle differentiation genes and was therefore expected to coregulate late-bound enhancers. To substantiate this further, we compared in vivo bound Mef2 enhancers, identified in our previous study (Sandmann et al. 2006), with the Biniou-bound enhancer regions at the same stages of development. In agreement with the motif enrichment, there is substantial combinatorial binding of Biniou and Mef2 on the early-bound and continuousbound enhancers: $65.1 \%$ and $50.4 \%$, respectively (Supplementary Fig. 6). In contrast, only $20.1 \%$ of the late Biniou-bound enhancers are cobound by Mef2. The same trend holds true in the other direction: There is no significant Biniou binding to many enhancers regulated by Mef2 at late developmental stages (e.g., the Mef2-bound enhancers for the contractile proteins $M h c, M l c 1$, and Mlc2) (Sandmann et al. 2006). This indicates that the VM may have two largely independent differentiation programs, one governed by Mef2 regulating more general muscle contractile proteins, and a second more VM-specific program driven by Biniou. 
Biniou consensus motifs are overrepresented in all three classes of temporal enhancers, providing global confirmation of the specificity of the ChIP-bound regions. Biniou motifs are particularly highly enriched in the continuous-bound and late-bound enhancers (Fig. 3). This highlights a prominent role for Biniou in regulating enhancer activity at late stages of VM development. The inability of Biniou to bind to the late enhancers at early stages of development implies a mechanism that either blocks Biniou binding to these CRMs early in development or enhances Biniou's binding later in development. This could be mediated by many different mechanisms. Binding of the C.elegans FoxA TF, PHA-4, to early versus late pharyngeal muscle enhancers is primarily determined by the presence of high or low affinity binding sites, respectively (Gaudet and Mango 2002). We detected no apparent differences in the Biniou motif between the early- and late-bound VM enhancers, and therefore favor a combinatorial model with as-yet-unidentified cofactors. This is strongly supported by the restricted expression of all Biniou-bound CRMs examined, necessitating extensive combinatorial regulation to limit their activity.

\section{Combinatorial binding to early VM enhancers by Bagpipe and Biniou}

The specific enrichment of Bagpipe motifs in Biniou early-bound CRMs, in addition to the similarity of bagpipe and biniou mutant phenotypes, implies a potential for combinatorial regulation by these two TFs during the stages of VM specification.
As Biniou is downstream from Bagpipe, it has been very difficult to differentiate between a direct regulatory role by Bagpipe versus an indirect requirement via Biniou using genetic studies. To investigate the molecular function of bagpipe and its potential occupancy on Biniou-bound CRMs, we performed ChIP-on-chip experiments using anti-Bagpipe antibodies. As described for Biniou, these experiments were performed with four biological repeats using two independent anti-Bagpipe antisera. The ChIPs were performed at stages 10-11 (6-8 h), covering the time span when bagpipe is transiently expressed in the trunk VM. This experiment identified 80 Bagpipe-bound genomic regions, using the same criteria as the Biniou experiments (Supplementary Table 4).

A number of genomic regions are exclusively bound by Bagpipe, with no detectable Biniou binding at stages 10-11 of development (Fig. 4A,D, Group 3). For example, the Bagpipe-bound region within the intron of CG8503: This enhancer is sufficient to drive transient expression in the trunk VM at stages 10-11 (Fig. 4B, arrow), reflecting the transient expression of bagpipe in this tissue. Other Bagpipe-bound enhancer regions contain low levels of Biniou binding (below our significance cutoff). The slp1 enhancer is within this class. This region drives expression in the foregut VM (Fig. 4C, arrow), recapitulating the endogenous gene's expression. Together these enhancers demonstrate that Bagpipe provides a direct regulatory role within the VM developmental program, independently of Biniou.

In contrast, $51 \%$ of Bagpipe enhancers are cobound by Biniou at the same stage of development (Fig. 4A). This
Figure 4. Combinatorial binding of Bagpipe and Biniou during VM specification. (A) A Venn diagram showing the overlap of Bagpipe- and Biniou-bound enhancers at stages $10-11$ (6- to 8-h time period). Fiftyone percent of Bagpipe-bound CRMs are cobound by Biniou. Biniou regulates twice as many CRMs as Bagpipe during these stages of VM development. The majority of Bagpipe's regulation is mediated via combinatorial binding to a subset of Biniou-bound CRMs. $(B, C)$ In situ hybridization of reporter transcripts for two Bagpipe-bound enhancers. (B) The CG8503 CRM drives transient expression in the trunk VM at stage 11 (arrow), in addition to somatic muscle expression. (C) The slp1 enhancer drives specific expression in the foregut VM from stage 13 (arrow). (D) The Bagpipe-bound CRMs fall into three clusters, distinguished by their temporal occupancy of Biniou. Group 1 enhancers are cobound by Bagpipe and Biniou at stages $10-11$ and remain bound by Biniou throughout development, while Group 2 enhancers are transiently bound by Biniou at early stages. Group 3 enhancers are largely not bound by Biniou, and represent the only Bagpipe-specific CRMs identified. The levels of enrichment are indicated by the color scale $\left(\log _{2}\right)$.
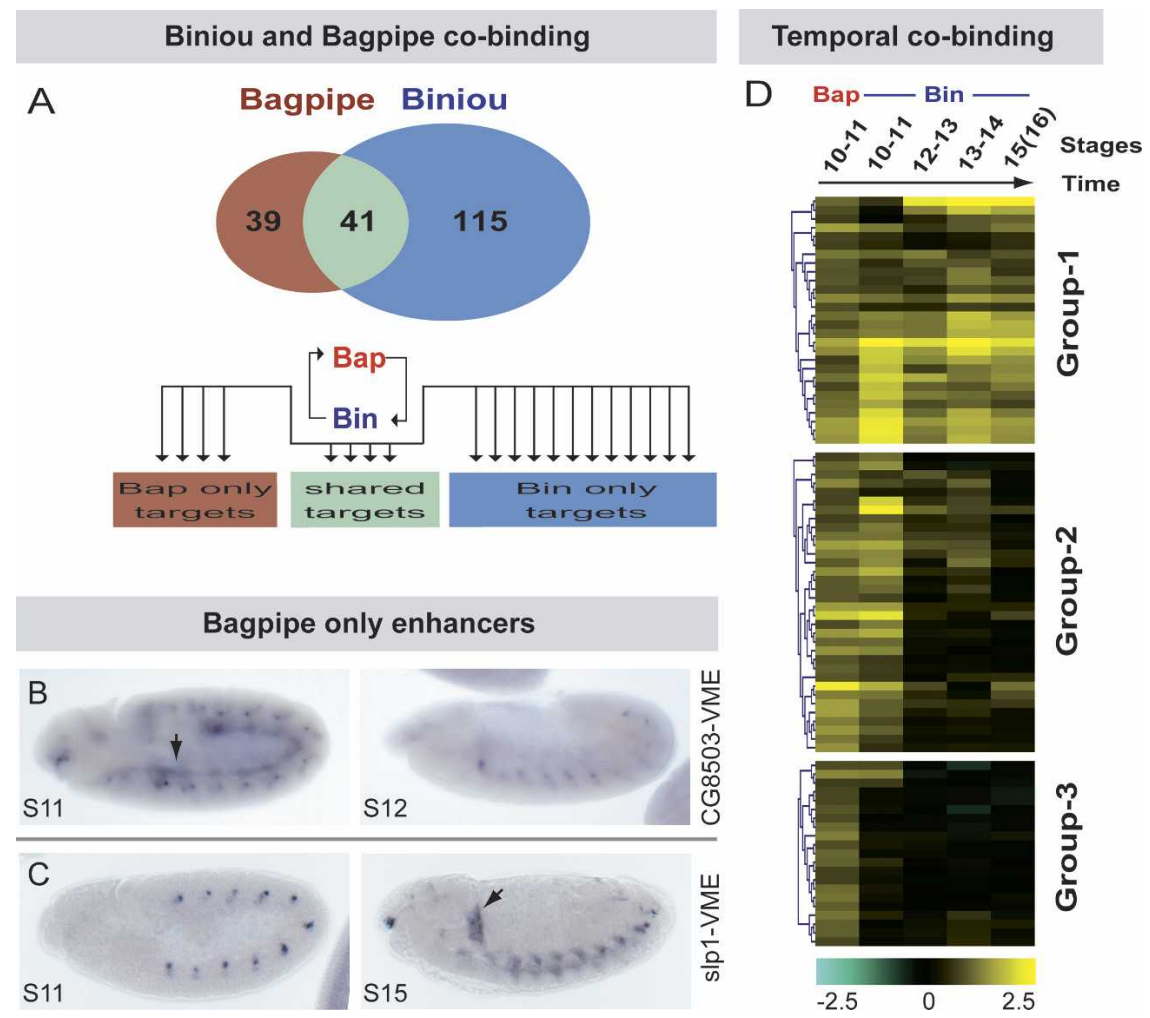
extensive combinatorial binding provides the first evidence of global coregulation by these TFs during early stages of VM specification. These cobound enhancers (Fig. 4A) suggests that transient Biniou occupancy on early group enhancers (Fig. 2A), may in part be due to cobinding with Bagpipe, which is transiently expressed at these stages. To investigate this we examined the temporal profile of Biniou binding to the 80 Bagpipe-bound CRMs using K-means clustering. Two distinct classes of Biniou-Bagpipe-cobound CRMs were apparent (Fig. 4D): Group 1 enhancers are cobound at stages 10-11 and remain continuously bound by Biniou at later developmental time points. This indicates that Biniou does not require the presence of Bagpipe to bind to the trunk VM enhancers among this class. In contrast, Group 2 enhancers are cobound by Biniou and Bagpipe at stages 10-11 of development, but are largely not bound by Biniou later in development (Fig. 4D). In the context of these early enhancers, Bagpipe binding may be the temporal cue dictating transient Biniou binding. Many of these CRMs are likely to be cooperatively regulated by both TFs.

\section{CRM binding reflects a regulatory requirement for target gene expression}

The extensive enhancer occupancy by Biniou suggests that this TF is required to regulate a comprehensive transcriptional program within the VM, both independently and in combination with Bagpipe. This is strongly supported by our global analysis of gene expression in biniou mutant embryos: A large number of genes have reduced expression during the first three time points examined (stages at which VM cells are still present in biniou mutant embryos), demonstrating that biniou is genetically required for their expression (Supplementary Fig. 1, cluster 1). To assess this further we performed a detailed analysis of the requirement of biniou for a number of target genes' expression in vivo and for enhancer activation in vitro.

In situ hybridization was performed on six Biniou direct target genes in both biniou loss of function mutant embryos and in transheterozygous deficiencies removing the bagpipe locus. mib2, CG17181, lmd, and salm are direct targets of both Biniou and Bagpipe via shared CRMs, while the otk and ken CRMs are only bound by Biniou. Due to the complete absence of trunk gut muscle in both mutants at late stages of development, the expression of all target genes in the trunk VM was examined at stage 11, when these cells are still present (Azpiazu and Frasch 1993; Zaffran et al. 2001).

Five of the examined direct target genes are specifically expressed in the primordia of the trunk VM (Fig. 5A-E, white arrowhead), three of which have additional expression within the hindgut and/or longitudinal VM domain (Fig. 5A-C, asterisk). The expression of all genes is severely diminished in the trunk VM in biniou or bagpipe mutant embryos, compared with wild-type

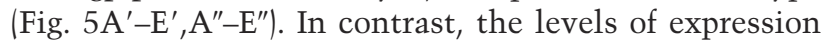
in the ectoderm (salm), nervous tissue (otk), and somatic muscle (mib2, CG17181), tissues where biniou and bagpipe are not expressed, remain unchanged (Fig. 5, white arrows). The mutual genetic interdependency of Biniou and Bagpipe in the trunk VM makes it difficult to definitively show a direct requirement for one of the TFs in this tissue in vivo. However, Biniou and Bagpipe are not dependent on each other for their expression in the foregut and hindgut VM (Zaffran et al. 2001), providing a useful system to confirm direct regulation by either TF. Three genes with enhancers bound by both TFs, mib2, CG17181, and Imd, are expressed in the hindgut VM (Fig. 5A-C, asterisk). All three genes have diminished hindgut VM expression in biniou and bagpipe mutant embryos (Fig. $5 \mathrm{~A}^{\prime}-\mathrm{C}^{\prime}, \mathrm{A}^{\prime \prime}-\mathrm{C}^{\prime \prime}$, asterisk), indicating a direct requirement for Biniou and Bagpipe to regulate their expression. This is particularly striking for mib2 and $1 \mathrm{md}$ (Fig. 5, asterisk, cf A,C and $\mathrm{A}^{\prime}, \mathrm{C}^{\prime}$ ). The sixth gene examined, ken, is expressed in the foregut VM from stage 14, as well as in the overlying ectoderm (Fig. 5F, asterisk and arrow, respectively). The foregut enhancer of ken (Fig. $2 \mathrm{H}, \mathrm{I}$ ) is specifically bound by Biniou and not by Bagpipe. The expression of this gene is severely reduced in biniou mutant embryos and is unaffected in bagpipe mutants (Fig. $\left.5 \mathrm{~F}^{\prime}-\mathrm{F}^{\prime \prime}\right)$, demonstrating direct regulation by Biniou, independent of bagpipe activity.

To further examine the ability of Biniou to regulate enhancer activity either independently or combinatorially with Bagpipe we used an in vitro transient luciferase-reporter assay. Cotransfection of increasing amounts of Biniou, Bagpipe, or a combination of both TFs was used to asses their ability to regulate enhancer activity in Drosophila Kc cells, a cell line of embryonic origin. All assays were performed in triplicate with six independent repeats to allow statistically analysis of enhancer activity.

Two enhancers that are cobound by Biniou and Bagpipe, the mib2 and CG17181 enhancers, were activated by Biniou transfection up to approximately twofold (Fig. 5G). As predicted from the in vivo binding data, coexpression of both Biniou and Bagpipe caused a significant synergistic activation of enhancer activity. These results, in combination with the reduced expression of mib2 and CG17181 in biniou and bagpipe mutant embryos (Fig. 5A,B), indicate cooperative regulation of these enhancers in vivo. In contrast, the otk enhancer, which is only bound by Biniou in vivo, was strongly activated by this TF in vitro (up to 6.1-fold), and was not synergistically activated by the addition of Bagpipe (Fig. 5G). These results indicate that the trunk VM expression of otk is largely regulated by Biniou, with little or no contribution from Bagpipe. The specificity of Biniou activation of these three enhancers is highlighted by Biniou's inability to regulate an enhancer that it does not bind to, the Bagpipe-bound CG8503 enhancer (Fig. 5G). This enhancer is highly activated by Mef2, demonstrating that the enhancer construct is functional (Fig. 5G).

Interestingly, Bagpipe expression alone was not sufficient to activate any enhancers tested in this in vitro assay, suggesting that Bagpipe driven regulation generally requires recruitment of additional coregulators not present in Kc cells. These results reveal an inherent difference between Biniou and Bagpipe as transcriptional 
Figure 5. Biniou and Bagpipe activity are essential for target gene expression. In situ hybridization of six Biniou direct target genes in wild-type embryos, biniou mutant embryos $\left(b_{i n}^{R 22}\right)$, and bagpipe mutant embryos [transheterozygotes of $D f(3 R) e^{F 1}$ and $\left.D f(3 R) e^{D 7} R e 28-58\right]$. In all panels, trunk VM is indicated by the white arrowhead and hindgut or foregut VM are indicated by the asterisk. $(A, B)$ mib-2 and CG17181 are expressed in the trunk VM, hindgut VM, and somatic muscle in wild-type embryos. The VM expression is severely reduced in biniou $\left(A^{\prime}, B^{\prime}\right)$ or bagpipe $\left(A^{\prime \prime}, B^{\prime \prime}\right)$ mutant embryos, while the somatic muscle expression remains unaffected (white arrows). (C) Imd expression in the trunk VM and hindgut $\mathrm{VM}$ is dramatically reduced in biniou $\left(C^{\prime}\right)$ and bagpipe $\left(C^{\prime \prime}\right)$ mutants. $(D, E)$ salm and otk are expressed in the trunk VM, as well as the ectoderm and nervous system, respectively. Only the VM expression is dependent on Biniou $\left(D^{\prime}, E^{\prime}\right)$ or Bagpipe $\left(D^{\prime \prime}, E^{\prime \prime}\right)$ activity, while the expression in the ectoderm and nervous system is similar to wild type (white arrows). (F) ken is expressed in the foregut VM (asterisk) and ectoderm (white arrow) in wild-type embryos. The VM expression is severely reduced in biniou mutant embryos $\left(F^{\prime}\right)$ but not in bagpipe mutants $\left(F^{\prime \prime}\right)$, consistent with the lack of Bagpipe binding to the ken enhancer. All pictures are lateral views of stage 11 embryos, with the exception of $D-D^{\prime \prime}$, which are dorsal views, and $F-F^{\prime \prime}$, which are lateral views of stage 14. $(G)$ Luciferase assays in Drosophila Kc cells show both independent and synergistic regulation of enhancers. For each assay, $1 \mathrm{ng}, 10 \mathrm{ng}$, or $100 \mathrm{ng}$ of Biniou, Bagpipe, or a combination of both transcriptions was cotransfected with the enhancer indicated. The two cobound enhancers, mib2 and CG17181, are activated by Biniou transfection and are synergistically activated by Biniou and Bagpipe cotransfection. The Biniou-only enhancer, otk, is signifi-

cantly regulated by Biniou, with no synergistic activation by Bagpipe. In contrast, Biniou cannot activate the Bagpipe-only enhancer, CG8503, which is highly activated by Mef2. Although the Bagpipe cDNA is clearly functional, demonstrated by the synergistic activation of the mib2 and CG17181, Bagpipe alone was not sufficient to activate any enhancer regions tested. $(\bullet \bullet) P<0.01$ by Student's $t$-test $(n=6)$.

activators: While Biniou is a potent activator with or without Bagpipe, Bagpipe has little activation potential by itself.

In summary, the in vivo binding of Biniou to enhancers independently or in combination with Bagpipe, its ability to activity enhancers alone and synergistically with Bagpipe, in addition to the in vivo requirement of biniou for many target genes' expression, demonstrate Biniou's prominent regulatory role within the transcriptional program driving VM myogenesis. Previous studies found no obvious hindgut or foregut defects in biniou and bagpipe mutant embryos (Azpiazu and Frasch 1993; Zaffran et al. 2001). However, our data show that a num- ber of Biniou-bound CRMs drive expression in the hindgut or foregut VM (fd64a-early [Fig. 2C], ken [Fig. 2H,I], bap-FH [Fig. $\left.\left.1 \mathrm{~F}^{\prime}\right]\right)$, and that many direct target genes have reduced expression in these tissues in biniou mutant embryos (Fig. 5). These results clearly demonstrate that Biniou directly contributes to the transcriptional network within the foregut and hindgut VM, and further extends the regulatory role of this TF to all subtypes of VM.

\section{Conservation in the FoxF regulatory circuitry from flies to mice}

The high conservation in the FoxF DNA-binding domain $164 \%$ amino acid identity between Biniou and 
FoxF) (Zaffran et al. 2001) and the similarity in their gut muscle phenotype suggests that these TFs may regulate an evolutionarily conserved set of direct target genes.

To test the regulatory conservation between Biniou and its target genes in vertebrates we examined the expression of three genes, for which we could identify a clear mouse ortholog: otk (Ptk7 in mouse), salm (Sall4), and HLH54F (Tcf21). In situ hybridization was performed using probes directed against the mouse transcripts in Foxf1 and Foxf2 mutant embryos (Fig. 6). As Foxf1 and Foxf2 are partially functionally redundant within the splanchnic mesoderm (Ormestad et al. 2004, 2006), target gene expression is expected to be only moderately reduced in either single mutant. Double null mutants cannot be generated as compound heterozygotes die at birth (Ormestad et al. 2006). Foxf1-1- mutant embryos die between embryonic day 9.5 (E9.5) and E10.5 (Mahlapuu et al. 2001b), while Foxf2 ${ }^{-/-}$mutants develop to term (Wang et al. 2003). We therefore examined the expression of the orthologs of Biniou target genes at E8.5 in Foxf1 $1^{-/}$and at E12.5-E13.5 in Foxf2 $2^{-/-}$embryos.

Murine FoxF genes are initially expressed in nascent mesoderm of the primitive streak and are maintained in lateral and extraembryonic mesoderm. Later their expression is confined to the splanchnic mesoderm and its derivatives, such as the mesenchyme of gut (Mahlapuu et al. 2001b; Ormestad et al. 2004). This broad expression is reflected in the expression of their target genes. In Drosophila, otk and salm are expressed in the primordia of the trunk VM just after specification (Fig. 5E,D). In mice, their orthologs, Ptk7 and Sall4, are expressed in newly formed mesoderm within the primitive streak area at the posterior end of the embryo (Fig. 6A,B; Kohlhase et al. 2002; Jung et al. 2004). Importantly, both genes have consistently lower levels of expression in Foxf1 homozygous mutant embryos compared with wild-type littermates at E8.5, in all mutant embryos ex-

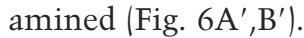

The HLH54F TF is specifically expressed in the Drosophila longitudinal VM (Georgias et al. 1997). The mouse ortholog, Tcf21 (also known as capsulin, epicardin, or Pod1) initiates expression in the heart at E8.5 (Lu et al. 1998). This expression remains unaltered in the FoxF2 knockout, consistent with the absence of FoxF expression in this tissue. At later stages, Tcf21 is expressed in the smooth muscle layer of the gastrointestinal and urogenital tracts (Fig. 6C). The expression in the gut mesenchyme is significantly reduced in Foxf2 mutant embryos (Fig. 6C').

Despite the expansion in FoxF TFs, our data indicate that at least some of their regulatory connections are conserved from flies to mice (Fig. 6D).

\section{Discussion}

To systematically dissect the transcriptional program driving VM development in vivo, we used two complementary genomic approaches: a time course of ChIP-onchip experiments and expression profiling of mutant em-

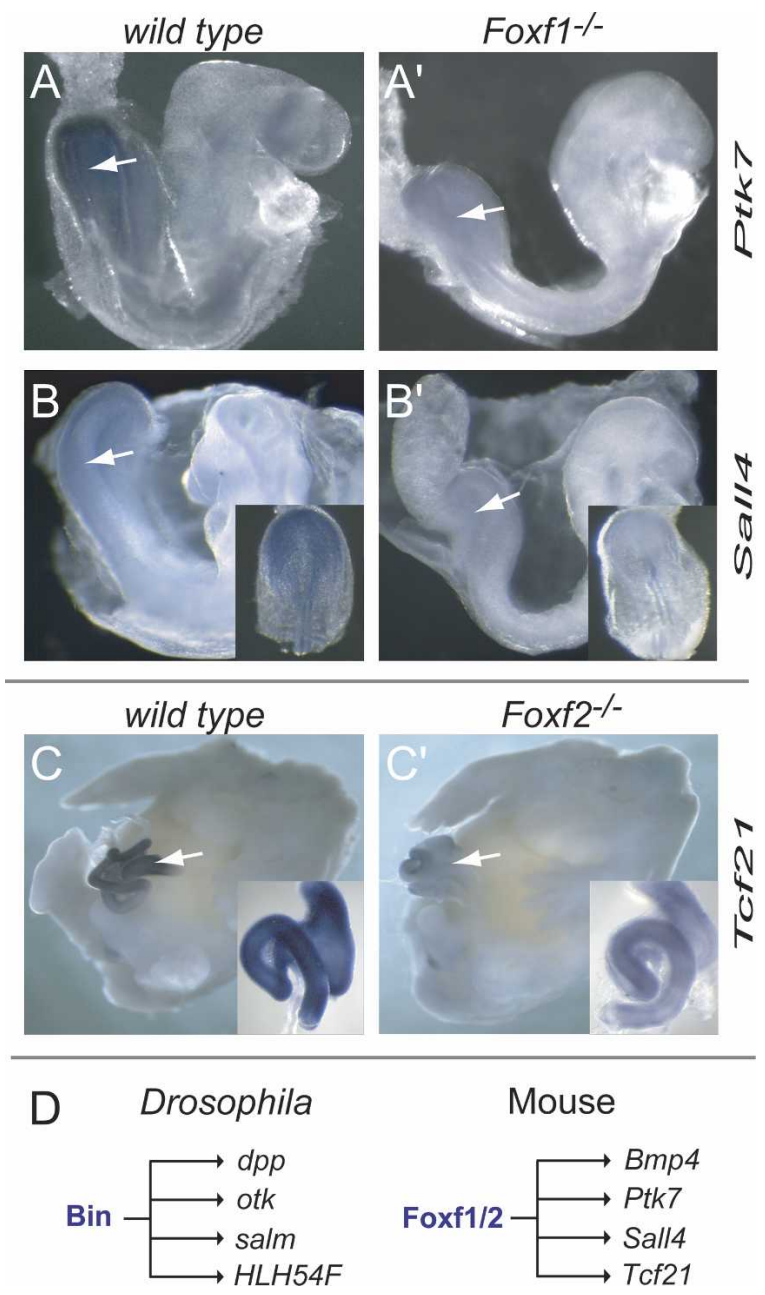

Figure 6. Conservation in the Biniou-FoxF regulatory circuitry from flies to mice. In situ hybridization in mouse embryos of the orthologous genes of three Drosophila Biniou direct targets. $\left(A, A^{\prime}\right)$ Lateral views of a wild-type embryo and a Foxf1 littermate at E8.5. The yolk sac was removed, while the allantois is intact. Ptk 7 (otk ortholog) expression in the primitive streak region $(A$, arrow) is markedly reduced in Foxf1 mutant embryos $\left(A^{\prime}\right) .\left(B, B^{\prime}\right)$ Sall4 (salm ortholog) is expressed in the primitive streak region $\left(B\right.$, arrow). $\left(B^{\prime}\right)$ FoxF1 mutant embryos have consistently lower primitive streak expression compared with wildtype littermates (arrow). The insets in $B$ and $B^{\prime}$ show the dorsal aspect of the primitive streak area of a wild-type and mutant embryo at an early stage of development, where the reduced expression in FoxF1-- is very apparent. $\left(C, C^{\prime}\right)$ In situ hybridization of whole embryos at E13.5. The strong expression of Tcf21 in the muscle of the herniated gut $(C)$ is strongly reduced in Foxf 2 mutant embryos $\left(C^{\prime}\right)$. The insets show a dissected posterior region of the small intestine, the caecum, and the proximal colon of wild type $(C)$ and Foxf $2^{-/-}\left(C^{\prime}\right)$, clearly displaying reduced Tcf21 expression in Foxf2 mutants. (D) Many of the regulatory connections between Binou and its target genes are conserved in mice, suggesting an ancient wiring of the VM regulatory network.

bryos performed during consecutive stages of embryogenesis. This global view revealed the following insights into the underlying cis-regulatory network: 
(1) Biniou binds to enhancers in a temporally regulated manner. As Biniou is expressed from VM specification until the end of development, this demonstrates that additional regulatory inputs are necessary to restrict Biniou activity. For the early-bound enhancers, some temporal specificity likely stems from combinatorial binding with Bagpipe. However, other TFs are also likely to be involved.

(2) Biniou-bound CRMs drive expression in diverse subtypes of VM. This restricted spatial expression again necessitates combinatorial regulation with additional factors. We propose that much of this spatial specificity is conferred through Biniou-mediated feed-forward regulation: Biniou regulates a large group of spatially restricted TFs and components of cell signaling pathways (Supplementary Fig. 7) that likely target different subsets of these CRMs. Such feed-forward regulation is a prevalent feature in many developmental networks (Davidson et al. 2002; Schroeder et al. 2004; Inoue et al. 2005; Koide et al. 2005; Sandmann et al. 2007a).

(3) The timing of Biniou enhancer occupancy is tightly correlated with the time span of enhancer activity. This is surprising given the extensive combinatorial binding necessary to produce restricted spatio-temporal expression of Biniou CRMs and suggests Biniou recruitment is the key trigger for enhancer activity. Taken together, our data indicate that Biniou provides VM enhancers with the competence to be expressed within the VM at the appropriate stage, and that these modules integrate extensive inputs from additional factors to restrict Biniou activity.

(4) Although bagpipe and biniou mutants phenocopy each other, their regulatory role within the underlying network is very different. The majority of Bagpipe's regulation occurs via combinatorial binding to BiniouBagpipe CRMs to regulate a shared set of target genes. From a limited number of enhancers assayed in vitro, Bagpipe's contribution to enhancer activity is mainly cooperative, with little regulatory potential by itself (Fig. $5 \mathrm{G})$. In contrast, Biniou targets an additional large group of CRMs during VM specification, and can regulate their activity independently of Bagpipe. This underlying nature of Biniou and Bagpipe's regulatory potential was not apparent from genetic studies due to the severity of their mutant phenotypes.

(5) Biniou provides regulatory input at all stages of VM development, not just specification. Moreover, the temporal regulation of target genes at either early or late stages reflects developmental progression. For example, $17 \%$ of target genes regulated late in development are involved in the formation or function of the neuromuscular junction, compared with $4 \%$ of continuously regulated targets and $0 \%$ of early targets (Supplementary Table 5). This reflects the requirement of neuronal stimulation for gut muscle contraction at the end of embryogenesis. Our results also revealed a new role for Biniou as a direct regulator of the transcriptional program within the foregut and hindgut VM.

(6) The underlying cis-regulatory circuitry between Biniou and its target genes is at least partially conserved from flies to mice. Four genes that are directly regulated by Biniou in flies require Fox $F$ function for their expression in mice. Due to the limited number of characterized FoxF direct target genes in vertebrates, it is currently too early to determine if VM development represents an ancient trans-bilaterian kernel (Davidson 2006).

Taken together, our data indicate that Biniou serves as a universal regulator of VM: The broad expression of Biniou in all VM subtypes and its extensive occupancy on VM enhancers strongly suggests that Biniou provides all VM cells, regardless of their origin or ultimate cell fate, with their VM identity.

\section{Materials and methods}

Expression profiling of biniou mutant embryos

A biniou loss-of-function allele ( bin $^{11}$ ) (Zaffran et al. 2001) was outcrossed to remove spurious mutations potentially maintained by the balancer chromosome. Pure populations of homozygous mutant embryos were obtained as described previously (Furlong et al. 2001a,b). Embryos ectopically expressing Biniou were collected from transheterozygote parents with twist-24BGal4 and UAS-biniou. One-hour collections of mutant and wild-type (Canton S) embryos were obtained and processed in parallel. The embryo staging was verified to ensure that mutant and wild-type embryos were tightly stage-matched. For each developmental time point, four independent embryo collections and hybridizations were performed. RNA amplification, labeling, and hybridization was conducted as described previously (Sandmann et al. 2006). The microarrays used for this study contain one 60-mer oligonucleotide for every predicted Drosophila gene (INDAC design; for details see FlyMine).

\section{ChIPs}

ChIPs were performed as described previously (Sandmann et al. 2007b). ChIPs were conducted with two independent antibodies for each TF. For Biniou, both antisera were generated in this study, raised against amino acids 135-614. For Bagpipe, we used one antibody generated in this study (raised against amino acids 1-382), and a second antibody generously donated by M. Frasch (University of Erlangen-Nuremberg, Erlangen, Germany). For each developmental time period, four independent embryo collections, chromatin preparations, and immunoprecipitations were obtained. Both immunoprecipitations and mock reactions were hybridized against a genomic reference sample, using appropriate dye swap controls to minimize dye biases.

\section{Analysis of array data and accession numbers}

The raw data from both tiling and cDNA arrays were normalized using print-tip combined with LOWESS normalization. To identify differentially expressed genes in biniou mutant embryos, the biniou mutant samples were hybridized directly against the stage-matched wild-type samples. A one-class SAM analysis was performed on four independent biological repeats per time point, using Mev (Saeed et al. 2003). Genes with a $q<0.01$ and a fold change of $\log _{2}>0.7$ or less than -0.7 at one or more developmental time points were considered differentially expressed.

To identify ChIP-enriched fragments, the experimental samples were hybridized against genomic DNA. The four mock/genomic DNA and four ChIP/genomic DNA ratios were compared in a two-class paired SAM analysis. Only genomic regions with a $q<0.01$ and a fold enrichment $>0.7\left(\log _{2}\right)$ were considered 
to be significantly bound. All microarray data are available from ArrayExpress (accession nos. E-TABM-249, E-TABM-247, E-TABM-250, E-TABM-251, and A-MEXP-718), or alternatively at http://furlonglab.embl.de/data.

Biniou and Bagpipe binding and mutant expression data can be visualized using a searchable Web site at http://furlonglab. embl.de/data/browse_chip_mod.

\section{Generation of transgenic reporter strains}

Genomic fragments of the following coordinates were cloned into the $\mathrm{pH}$-Stinger vector (Barolo et al. 2000) for germline transformation: chr3R: 2302000-2302930 (CG2330-VME); chr3R: 18854362-18855948 (lmd-VME); chr3R: 17218144-17220402 (bap-FH-VME); chr2L: 16482002-16483204 (dac-VME); chr3R: 27528340-27531000 (ttk-VME); chr3L: 4000681-4002080 (fd64aeVME); chr2R: 13274315-13275818 (HLH54F-VME); chr2R: 7524515-7526750 (otk-VME); chr2L: 19031758-19033780 (mib2-VME); chr3L: 3997574-3999422 (fd64a ${ }^{-1-} \mathrm{VME}$ ); chr2R: 19379642-19381101 (ken-VME); chr2R: 9758088-9758850 (CG8503-VME); chr2L: 3813750-3815714 (based on Genome release 4.2). For all enhancers, with the exception of ken, at least two independent transgenic lines were obtained and assayed.

\section{In situ hybridization}

In situ hybridizations in Drosophila embryos were done using standard protocols and as described previously (Furlong et al. 2001a). The following ESTs were used to generate digoxigeninlabeled probes: GH28686 (mib2), RH55216 (CG17181), LD47926 (lmd), LD17463 (salm), LP21473 (otk), GM09101 (ttk), GH12495 (ken), RE64054 (dac), and LD42676 (CG2330). The probe directed against the GFP transcript was made by amplifying the GFP sequence from the pH-Stinger vector. bin cDNA /gift from $M$. Frasch) was used to generate a Biotin-labeled bin probe. Double in situs were performed by using $\alpha$-DIG-POD and $\alpha$-Biotin-POD (Roche) antibodies and were detected sequentially with Cy3 and Cy5 (PerkinElmer TSA kit).

Mouse embryos were collected from $\mathrm{C} 57 \mathrm{Bl} / 6$ pregnant females at E8.5 (Foxf1 mutant) or E13.5 (Foxf2 mutant). Homozygous mutants and wild-type littermates were identified by PCR genotyping, as described previously (Mahlapuu et al. $2001 \mathrm{~b}$; Wang et al. 2003). Embryos were fixed in $4 \%$ paraformaldehyde overnight and dehydrated in methanol. Wholemount in situ hybridization with digoxigenin-labeled riboprobes was performed with an InsituPro robot (Intavis AG), using a standard protocol (Rosen and Beddington 1994) with minor adjustments to fit the robotic format.

\section{Luciferase assays}

Drosophila Kc-cells were transiently transfected using standard protocols (Cellfectin, Invitrogen). TFs Biniou (cDNA from M. Frasch), Bagpipe (EST, RE13967), or Mef2 (EST GH 24,154), were expressed using the pAC5.1b vector (Invitrogen). Enhancer activity was assayed using the pGL3 luciferase reportor vector (Promega) modified with a hsp70 promotor (see transgenic reporter strains for enhancer coordinates). The total amount of DNA transfected was normalized by substituting with empty pAC5.1b vector. Luciferase assay was performed as described (Dualluciferase Reporter assay, Promega), employing a PerkinElmer 1420 Luminescence Counter. Categories were compared with a $t$-test.

\section{Acknowledgments}

We are very grateful to Asifa Akhtar, Detlev Arendt, Jürg Müller, Guillaume Junion, and Robert Zinzen for critically reading the manuscript. We thank Manfred Frasch for supplying Biniou cDNA and Bagpipe antibody. We thank all members of the Furlong laboratory, especially Charles Girardot for his help with BASE and motif analysis, Paulo Cunha for advice with the luciferase assay, and Ioannis Amarantos for his significant contribution to making the tiling arrays. We are grateful to Lars Jensen and Peer Bork for help with the target gene assignments. We thank Anne-Mari Voie and the MyoRes transgenic platform for embryo injections.

\section{References}

Azpiazu, N. and Frasch, M. 1993. tinman and bagpipe: Two homeo box genes that determine cell fates in the dorsal mesoderm of Drosophila. Genes \& Dev. 7: 1325-1340.

Barolo, S., Carver, L.A., and Posakony, J.W. 2000. GFP and $\beta$ galactosidase transformation vectors for promoter/enhancer analysis in Drosophila. Biotechniques 29: 726-732.

Baylies, M.K. and Michelson, A.M. 2001. Invertebrate myogenesis: Looking back to the future of muscle development. Curr. Opin. Genet. Dev. 11: 431-439.

Berkes, C.A. and Tapscott, S.J. 2005. MyoD and the transcriptional control of myogenesis. Semin. Cell Dev. Biol. 16: 585595.

Bitar, K.N. 2003. Function of gastrointestinal smooth muscle: From signaling to contractile proteins. Am. J. Med. 115 (Suppl. 3A): 15S-23S. doi: 10.1016/S0002-9343(03)00189-X.

Black, B.L. and Olson, E.N. 1998. Transcriptional control of muscle development by myocyte enhancer factor-2 (MEF2) proteins. Annu. Rev. Cell Dev. Biol. 14: 167-196.

Bodmer, R. 1993. The gene tinman is required for specification of the heart and visceral muscles in Drosophila. Development 118: 719-729.

Davidson, E. 2006. The regulatory genome: Gene regulatory networks in development and evolution. Academic Press, New York.

Davidson, E.H., Rast, J.P., Oliveri, P., Ransick, A., Calestani, C., Yuh, C.H., Minokawa, T., Amore, G., Hinman, V., ArenasMena, C., et al. 2002. A genomic regulatory network for development. Science 295: 1669-1678.

Dettman, R.W., Turner, F.R., and Raff, E.C. 1996. Genetic analysis of the Drosophila $\beta 3$-tubulin gene demonstrates that the microtubule cytoskeleton in the cells of the visceral mesoderm is required for morphogenesis of the midgut endoderm. Dev. Biol. 177: 117-135.

Doevendans, P.A. and van Bilsen, M. 1996. Transcription factors and the cardiac gene programme. Int. J. Biochem. Cell Biol. 28: 387-403.

Frasch, M. 1995. Induction of visceral and cardiac mesoderm by ectodermal Dpp in the early Drosophila embryo. Nature 374: 464-467.

Furlong, E.E., Andersen, E.C., Null, B., White, K.P., and Scott, M.P. 2001a. Patterns of gene expression during Drosophila mesoderm development. Science 293: 1629-1633.

Furlong, E.E., Profitt, D., and Scott, M.P. 2001b. Automated sorting of live transgenic embryos. Nat. Biotechnol. 19: 153156.

Gaudet, J. and Mango, S.E. 2002. Regulation of organogenesis by the Caenorhabditis elegans FoxA protein PHA-4. Science 295: 821-825.

Georgias, C., Wasser, M., and Hinz, U. 1997. A basic-helixloop-helix protein expressed in precursors of Drosophila longitudinal visceral muscles. Mech. Dev. 69: 115-124.

Goldstein, M.A. and Burdette, W.J. 1971. Striated visceral muscle of Drosophila melanogaster. J. Morphol. 134: 315334. 
Inoue, T., Wang, M., Ririe, T.O., Fernandes, J.S., and Sternberg, P.W. 2005. Transcriptional network underlying Caenorhabditis elegans vulval development. Proc. Nat1. Acad. Sci. 102: 4972-4977.

Jung, J.W., Shin, W.S., Song, J., and Lee, S.T. 2004. Cloning and characterization of the full-length mouse Ptk7 cDNA encoding a defective receptor protein tyrosine kinase. Gene 328: 75-84.

Kohlhase, J., Heinrich, M., Liebers, M., Frohlich Archangelo, L., Reardon, W., and Kispert, A. 2002. Cloning and expression analysis of SALL4, the murine homologue of the gene mutated in Okihiro syndrome. Cytogenet. Genome Res. 98: 274-277.

Koide, T., Hayata, T., and Cho, K.W. 2005. Xenopus as a model system to study transcriptional regulatory networks. Proc. Nat1. Acad. Sci. 102: 4943-4948.

Kumar, J.P. and Moses, K. 2001. Expression of evolutionarily conserved eye specification genes during Drosophila embryogenesis. Dev. Genes Evol. 211: 406-414.

Kusch, T. and Reuter, R. 1999. Functions for Drosophila brachyenteron and forkhead in mesoderm specification and cell signalling. Development 126: 3991-4003.

Lamey, T.M., Koenders, A., and Ziman, M. 2004. Pax genes in myogenesis: Alternate transcripts add complexity. Histol. Histopathol. 19: 1289-1300.

Lee, H.H. and Frasch, M. 2000. Wingless effects mesoderm patterning and ectoderm segmentation events via induction of its downstream target sloppy paired. Development 127: 5497-5508.

Lee, H.H. and Frasch, M. 2005. Nuclear integration of positive Dpp signals, antagonistic Wg inputs and mesodermal competence factors during Drosophila visceral mesoderm induction. Development 132: 1429-1442.

Lee, H.-H., Zaffran, S., and Frasch, M. 2006. Develpment of the larval visceral musculature. In Muscle development in Drosophila (ed. H. Sink), pp. 62-78. Landes Bioscience, Austin, TX.

Lettice, L.A., Purdie, L.A., Carlson, G.J., Kilanowski, F., Dorin, J., and Hill, R.E. 1999. The mouse bagpipe gene controls development of axial skeleton, skull, and spleen. Proc. Natl. Acad. Sci. 96: 9695-9700.

Lu, J., Richardson, J.A., and Olson, E.N. 1998. Capsulin: A novel bHLH transcription factor expressed in epicardial progenitors and mesenchyme of visceral organs. Mech. Dev. 73: 23-32.

Mahlapuu, M., Enerback, S., and Carlsson, P. 2001a. Haploinsufficiency of the forkhead gene Foxf1, a target for sonic hedgehog signaling, causes lung and foregut malformations. Development 128: 2397-2406.

Mahlapuu, M., Ormestad, M., Enerback, S., and Carlsson, P. $2001 \mathrm{~b}$. The forkhead transcription factor Foxf1 is required for differentiation of extra-embryonic and lateral plate mesoderm. Development 128: 155-166.

Mathies, L.D., Kerridge, S., and Scott, M.P. 1994. Role of the teashirt gene in Drosophila midgut morphogenesis: Secreted proteins mediate the action of homeotic genes. Development 120: 2799-2809.

Ormestad, M., Astorga, J., and Carlsson, P. 2004. Differences in the embryonic expression patterns of mouse Foxf1 and -2 match their distinct mutant phenotypes. Dev. Dyn. 229: 328-333.

Ormestad, M., Astorga, J., Landgren, H., Wang, T., Johansson, B.R., Miura, N., and Carlsson, P. 2006. Foxf1 and Foxf2 control murine gut development by limiting mesenchymal Wnt signaling and promoting extracellular matrix production. Development 133: 833-843.

Riechmann, V., Irion, U., Wilson, R., Grosskortenhaus, R., and
Leptin, M. 1997. Control of cell fates and segmentation in the Drosophila mesoderm. Development 124: 2915-2922.

Rosen, B. and Beddington, R. 1994. Detection of mRNA in whole mounts of mouse embryos using digoxigenin riboprobes. Methods Mol. Biol. 28: 201-208.

Saeed, A.I., Sharov, V., White, J., Li, J., Liang, W., Bhagabati, N., Braisted, J., Klapa, M., Currier, T., Thiagarajan, M., et al. 2003. TM4: A free, open-source system for microarray data management and analysis. Biotechniques 34: 374-378.

San Martin, B. and Bate, M. 2001. Hindgut visceral mesoderm requires an ectodermal template for normal development in Drosophila. Development 128: 233-242.

Sandmann, T., Jensen, L.J., Jakobsen, J.S., Karzynski, M.M., Eichenlaub, M.P., Bork, P., and Furlong, E.E. 2006. A temporal map of transcription factor activity: mef2 directly regulates target genes at all stages of muscle development. Dev. Cell 10: 797-807.

Sandmann, T., Girardot, C., Brehme, M., Tongprasit, W., Stolc, V., and Furlong, E.E. 2007a. A core transcriptional network for early mesoderm development in Drosophila melanogaster. Genes \& Dev. 21: 436-449.

Sandmann, T., Jakobsen, J.S., and Furlong, E.E. 2007b. ChIP-onchip protocol for genome-wide analysis of transcription factor binding in Drosophila melanogaster embryos. Nat. Protoc. 6: 2839-2855.

Schroeder, M.D., Pearce, M., Fak, J., Fan, H., Unnerstall, U., Emberly, E., Rajewsky, N., Siggia, E.D., and Gaul, U. 2004. Transcriptional control in the segmentation gene network of Drosophila. PLoS Biol. 2: E271. doi: 10.1371/journal.pbio. 0020271.

Tseng, H.T., Shah, R., and Jamrich, M. 2004. Function and regulation of FoxF1 during Xenopus gut development. Development 131: 3637-3647.

Wang, T., Tamakoshi, T., Uezato, T., Shu, F., Kanzaki-Kato, N., Fu, Y., Koseki, H., Yoshida, N., Sugiyama, T., and Miura, N. 2003. Forkhead transcription factor Foxf2 (LUN)-deficient mice exhibit abnormal development of secondary palate. Dev. Biol. 259: 83-94.

Zaffran, S. and Frasch, M. 2002. The $\beta 3$ tubulin gene is a direct target of bagpipe and biniou in the visceral mesoderm of Drosophila. Mech. Dev. 114: 85-93.

Zaffran, S., Kuchler, A., Lee, H.H., and Frasch, M. 2001. biniou (FoxF), a central component in a regulatory network controlling visceral mesoderm development and midgut morphogenesis in Drosophila. Genes \& Dev. 15: 2900-2915. 


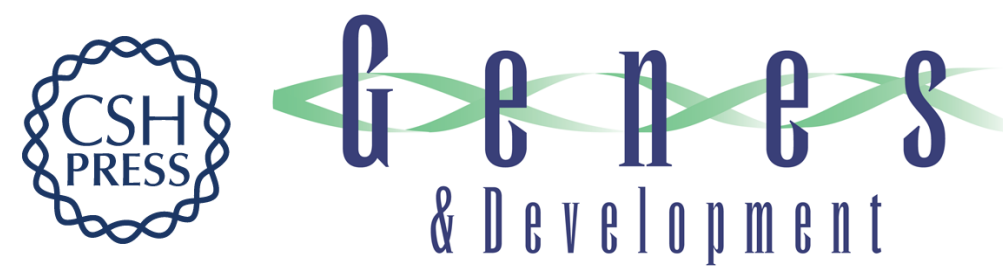

\section{Temporal ChIP-on-chip reveals Biniou as a universal regulator of the visceral muscle transcriptional network}

Janus S. Jakobsen, Martina Braun, Jeanette Astorga, et al.

Genes Dev. 2007, 21:

Access the most recent version at doi:10.1101/gad.437607

Supplemental
Material http://genesdev.cshlp.org/content/suppl/2007/10/01/21.19.2448.DC1

References This article cites 44 articles, 19 of which can be accessed free at:

http://genesdev.cshlp.org/content/21/19/2448.full.html\#ref-list-1

License

Email Alerting

Receive free email alerts when new articles cite this article - sign up in the box at the top

Service

right corner of the article or click here.

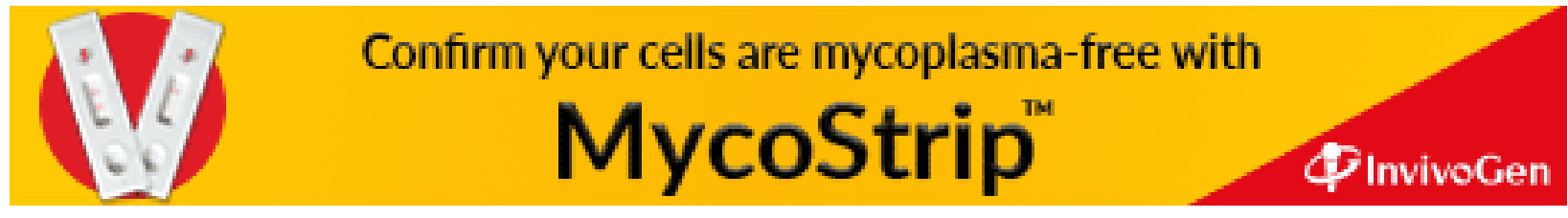

